Artículo

\title{
Impacto de la inclusión de información extranjera sobre la evaluación genética mexicana de sementales Holstein
}

\author{
Gustavo Javier Martínez Marín ${ }^{a}$ \\ Felipe de Jesús Ruiz López ${ }^{\text {a }}$ \\ Carlos Gustavo Vásquez Peláez ${ }^{\text {b }}$ \\ Sergio Iván Román Ponce ${ }^{a}$ \\ Adriana García Ruiz ${ }^{\mathrm{a}^{*}}$
}

\begin{abstract}
${ }^{\text {a }}$ Instituto Nacional de Investigaciones Forestales, Agrícolas y Pecuarias. Centro Nacional de Investigación Disciplinaria en Fisiología y Mejoramiento Animal. Km 1 carretera Ajuchitlán-Colón, Ajuchitlán, Querétaro, México.

${ }^{\mathrm{b}}$ Universidad Nacional Autónoma de México, Facultad de Medicina Veterinaria y Zootecnia, Ciudad de México, México.
\end{abstract}

*Autor de correspondencia: garcia.adriana@inifap.gob.mx

\section{Resumen:}

El objetivo del presente estudio fue evaluar el impacto de la inclusión de información extranjera de sementales de la raza Holstein, sobre su evaluación genética para las características de producción de leche (PL), grasa (PG) y proteína (PP) en kilogramos. Lo anterior, se logró mediante la comparación de valores genéticos (VG) y confiabilidades (CF), agrupación de sementales por número de hijas, ordenamiento de los sementales y la superioridad genética esperada por año $\left(\Delta I_{s} / y s\right)$, con diferentes escenarios determinados por la intensidad de selección ( $i p_{s}$ ) de sementales usados en la evaluación genética nacional (EG$\mathrm{MEX}$ ), los incorporados a la evaluación genética internacional con hijas en México (EG-I) y la evaluación genética internacional con o sin hijas en México (EG-MACE). En total, se analizó la información de 5,825 sementales en la para PL y 3,914 para PG y PP. El impacto 
de la información extranjera en la EG-MEX es positivo, debido a que mejora la CF de los VG de los sementales usados en México. También, permitió observar diferencias importantes de los VG y CF entre las evaluaciones, generando una oportunidad de mejoramiento en la población Holstein mexicana; por lo que, es recomendable continuar con la participación en el programa de Interbull, y considerar el uso de la información validada de manera internacional en los procesos de selección de animales productores de leche y sus componentes. Estas acciones pueden contribuir de manera importante en el incremento del progreso genético productivo a nivel nacional.

Palabras clave: Valor genético, Confiabilidad, Evaluación genética, Correlación genetica.

Recibido: 14/05/2019

Aceptado: 22/11/2019

\section{Introducción}

La evaluación genética consiste en la predicción del mérito o valor genético (VG) de un animal considerando la información genealógica, fenotípica (registros de producción y componentes de la leche, conteo de células somáticas, y conformación, entre otras) ${ }^{(1)}$. La primera evaluación genética del ganado Holstein en México, fue realizada en 1974, por la Universidad de Guelph (Ontario, Canadá) a solicitud de la Asociación Holstein de México (AHM), incluyendo solamente características de conformación. Durante las siguientes décadas, se integraron características de producción de leche (PL), grasa (PG), proteína (PP) en kilogramos, entre otras, y se realizaron modificaciones en el sistema de calificación para la identificación de animales altamente productivos y funcionales que eran utilizados en nuestro país ${ }^{(1,2,3)}$. Actualmente, la AHM junto con el Centro Nacional de Investigación Disciplinaria en Fisiología y Mejoramiento Animal (CENID-FyMA) perteneciente al Instituto Nacional de Investigaciones Forestales, Agrícolas y Pecuarias (INIFAP), son los encargados de realizar cada cuatro meses las evaluaciones genéticas para producción y calidad de leche, 27 caracteres de conformación, longevidad, células somáticas y los índices de selección de ganado Holstein registrado ante la AHM. La metodología utilizada para la predicción de estos VG, es la del Mejor Predictor Lineal Insesgado (BLUP), que consiste en un modelo mixto que engloba ecuaciones simultaneas de efectos fijos y aleatorios para realizar la estimación de componentes de varianza, basado en el rendimiento individual y en la representación de todas las relaciones genéticas entre los individuos ${ }^{(4)}$. Los resultados de estas evaluaciones, sirven como identificadores de los mejores ejemplares, proporcionando diversas opciones a los ganaderos para los procesos de selección. 
A medida que aumenta el intercambio de material genético alrededor del mundo, existe la posibilidad de que un mismo animal tenga descendencia en diferentes países y, por lo tanto, cuente con más de una evaluación genética. En el caso de México, un alto porcentaje de material genético es importado en forma de semen congelado y para cuando los sementales importados tienen información en México para la predicción de su VG, estos ya cuentan con una evaluación genética en su país de orígen con más información que la generada en nuestro país. Ante la inquietud de incorporar la información generada a nivel global, Schaeffer ${ }^{(5)}$ propuso la metodología de evaluación genética que integra información de múltiples países (MACE). Para lograr lo anterior, se construyen ecuaciones de regresión con el objeto de predecir los VG de los sementales importados en la base del país importador, ajustando según el origen de la información ${ }^{(6,7)}$. Existen numerosas ventajas de utilizar el sistema MACE, entre las cuales destacan: 1) el rendimiento de sementales ajustado por la información de producción de las hijas provenientes de las diversas evaluaciones de los países participantes y; 2) la identificación de ejemplares existentes en otros países que puedan ser mejoradores bajo las condiciones propias del país importador ${ }^{(5,7)}$.

El objetivo del presente estudio fue evaluar el impacto de la inclusión de información extranjera de sementales de la raza Holstein sobre su evaluación genética para las características de PL, PG y PP, y predecir las tasas de mejoramiento genético esperadas de la utilización de las evaluaciones resultantes. Con la incorporación de dicha información, se espera mejorar la precisión de los VG y por ende, las tasas de mejoramiento genético de las características evaluadas.

\section{Material y métodos}

\section{Base de datos}

Los registros productivos de leche y sus componentes se recopilaron por el sistema de control de producción oficial de la AHM y procesados por el CENID-FyMA-INIFAP. Para el cálculo de VG de la evaluación genética mexicana (EG-MEX) se utilizó la información de 623,207 registros de PL, correspondientes a 355,786 animales provenientes de 527 hatos y 193,236 registros para PG y PP, correspondientes a 103,829 animales provenientes de 167 hatos. Los establos de los cuales se obtuvo la información, se encuentran distribuidos en 17 diferentes entidades federativas de la República Mexicana. La información genealógica constó de 403,817 animales, incluyendo registros de hasta cinco generaciones para los animales con producción disponible. Los registros con información productiva fuera de los parámetros biológicos observados en el sistema de producción y en la raza (PL ajustada a 305 días $<3,500$ $\mathrm{kg} \mathrm{o}>22,000 \mathrm{~kg}$ por lactación, PG y PP $<1.5 \%$ o $>8.0 \%)^{(1)}$ fueron eliminados del estudio. 


\section{Estimación de los valores genéticos y confiabilidades}

El proceso de estimación de VG de la EG-MEX, se realizó por medio de un modelo animal de repetibilidad ${ }^{(1,8)}$, usando el software BLUPF90 con la paquetería SS-BLUPF90 ${ }^{(9,10)}$. El modelo utilizado para cada una de las tres características de producción ${ }^{(11)}$, fue el siguiente:

donde:

$$
y=X \beta+Z_{1} u+Z_{2} p+e
$$

$\boldsymbol{y}=$ vector de los registros productivos ajustados a 305 días y equivalente de madurez (leche, grasa o proteína respectivamente);

$\boldsymbol{\beta}=$ vector de efectos fijos (hato-año-estación, interacción semental-hato y edad al parto);

$\boldsymbol{X}=$ matriz de incidencia relacionada con los efectos fijos;

$Z_{1}=$ matriz de incidencia relacionada con los efectos aleatorios de $u$;

$\boldsymbol{u}=$ vector aleatorio de efectos genéticos aditivos;

$Z_{2}=$ matriz de incidencia relacionada con efectos aleatorios de ambiente permanente;

$\boldsymbol{p}=$ vector de efectos de ambiente permanente;

$\boldsymbol{e}=$ vector de efectos residuales.

Conjuntamente a la estimación de VG de los animales, se calculó su confiabilidad ${ }^{(8,12)}$ como:

$$
\left[1-\left(\frac{P E V}{\sigma_{E}^{2}}\right)(\lambda)\right]
$$

donde: $\sigma_{E}^{2}=$ Varianza ambiental; $\lambda=$ la proporción de la varianza ambiental $\left(\sigma_{E}^{2}\right)$ entre la varianza genética aditiva $\left(\sigma_{A}^{2}\right)$ que es igual a $\left[\left(1-h^{2}\right) / h^{2}\right] ; P E V=$ Varianza del error de predicción, estimado como:

$$
P E V=\left(1-r_{A \hat{A}}^{2}\right) \sigma_{A}^{2}
$$

donde: $r_{A \widehat{A}}^{2}=$ Coeficiente de correlación entre el VG predicho y VG verdadero; $\sigma_{A}^{2}=$ varianza aditiva.

Durante este proceso, se estimaron los VG y las CF de 7,489 sementales para PL y de 5,148 para PG y PP. Dicha información fue proporcionada a Servicio Internacional de Evaluación de Sementales (Interbull) para su integración al sistema de EG-I.

\section{Evaluación genética internacional}

La información de VG y CF proveniente de la EG-MEX (7,489 sementales para PL y 5,148 para PG y PP), fue procesada bajo los lineamientos establecidos por el Área de Intercambio de Información Interbull (IDEA, por sus siglas en inglés), para su validación e incorporación a la evaluación genética internacional (EG-I) ${ }^{(7)}$. La información de cada uno de los países participantes es recopilada y ajustada por Interbull con el procedimiento MACE con base en la cantidad, la estructura de los grupos contemporáneos y las correlaciones internacionales ${ }^{(13,14)}$. Del total de la información proporcionada por México, 5,825 
sementales para PL y 3,914 para PG y PP contaron con información productiva de al menos una hija en más de un país, por lo que pudieron ser incorporados a la base productiva y de pedigrí a nivel internacional. Conjuntamente al proceso de EG-I, se realizó la prueba de correlaciones genéticas internacionales (CGI), en la cual, solo se integraron por país participante a aquellos sementales que cumplieron con los requisitos mínimos de prueba especificados por Interbull ${ }^{(7,15)}$. México aportó información de 553 sementales para PL, 183 para PG y 181 para PP, para dicha prueba.

\section{Comparación de los valores genéticos y sus confiabilidades entre las evaluaciones genéticas nacional e internacional}

Las tendencias genéticas se analizaron a partir de los VG promedio obtenidos tanto en la EG-MEX como en la EG-I por año de nacimiento de los sementales. Con el objetivo de evaluar si el impacto de la información extranjera sobre la EG-MEX está relacionada con el número de hijas del semental en México, a cada semental se le asignó un grupo (más de 50, entre 10 y 50 y menos de 10 hijas) y se calcularon las medias y desviaciones estándar de VG y CF para cada grupo y evaluacion genética (EG-MEX y EG-I). Adicionalmente, para evaluar la concordancia entre las evaluaciones, se calcularon correlaciones de Pearson $(\rho \mathrm{P})$ tanto para VG como para CF de las tres características (PL, PG y PP). También, se calculó la tasa de mejoramiento genético esperado $(\beta)$ de la utilización de las EG-MEX y EG-I en la elección de sementales a través de un análisis de regresión lineal.

Posteriormente, se ordenaron a los sementales de forma descendente por su VG tanto para la EG-MEX como para la EG-I y fueron clasificados en diferentes escenarios de selección, tomando en cuenta los percentiles ( $i p_{s}=$ el mejor $10 \%, 20 \%, 30 \%, 40 \%$ y $50 \%$ ), para con ello, realizar las comparaciones de medias para VG y CF de las tres características (PL, PG y PP) por grupo, del número en común de sementales en ambas evaluaciones durante el ordenamiento, su asociación monótona por medio de la correlación de Spearman $(\rho S)$ y el cálculo de la superioridad genética esperada de los sementales por año $\left(\Delta I_{s} / y s\right)$ por el método descrito por Bourdon ${ }^{(16)}$ como:

$$
\Delta I_{s} / y s=i p_{s} r_{s} \sigma_{A} / L_{s}
$$

donde, $i p_{s}=$ intensidad de selección definida por el porcentaje de sementales genotípicamente superiores $(10 \%, 20 \%, 30 \%, 40 \%$ y $50 \%), r_{s}=$ precisión de los VG de los sementales, $\sigma_{A}=$ desviación genética aditiva de los sementales, y $L_{S}=$ Intervalo generacional de 6 años para sementales padres de $\operatorname{vacas}^{(17)}$.

Para cada uno de los escenarios de selección por percentiles, se calculó la $\Delta I_{S} / y s$ tomando las diferentes $i p_{s}$, pero con los resultados de la EG-MEX, EG-I y la evaluación genética internacional completa (EG-MACE), esta última haciendo referencia a todos los sementales 
participantes en la prueba internacional (150,300 sementales) ordenados de manera decendente y que pueden o no tener hijas en México.

\section{Resultados y discusión}

Los VG obtenidos en la prueba difieren en promedio $120 \mathrm{~kg}$ en PL (de -200 $\pm 315 \mathrm{~kg}$ en EGMEX a $-80.1 \pm 411.5 \mathrm{~kg}$ para EG-I), 4.6 para PG (de $-2.4 \pm 8.2 \mathrm{~kg}$ en EG-MEX a $2.2 \pm 12.5$ para EG-I) y 5.8 para PP (-3.6 $\pm 7.9 \mathrm{~kg}$ en EG-MEX a $2.2 \pm 12.3 \mathrm{~kg}$ para EG-I). Las CF en promedio son mayores en la EG-I que en EG-MEX, e incluso llegan a ser el doble (de 73 a 30 para PL y de 74 a 29 para PG y PP) al considerar información de hijas en producción en los diversos países participantes.

En ambas evaluaciones (EG-MEX y EG-I) se observó una tendencia positiva en el mejoramiento genético promedio por año de nacimiento resultando la EG-I con los mayores VG durante todo el periodo de estudio (Figura 1). También, se muestra un descenso de la CF promedio en los últimos años de estudio, evento que coincide con la disminución del número de sementales nacidos en los últimos años (Figura 2). El decenso de las CF puede ser explicada por la menor cantidad de hijas de sementales jóvenes evaluadas ${ }^{(18,19)}$. Un estudio previo sugiere que la disminución de los promedios de los VG pueden ser debido a que en un inicio todos los sementales son probados a través de la evaluación de algunas crías y una vez que se identifican a los mejores ejemplares, se utilizan de manera frecuente para incrementar el promedio de las tendencias genéticas dentro de la población y el número de hijas por semental ${ }^{(20)}$, efecto que puede ayudar a aumentar las similitudes de los VG calculados con las dos evaluaciones utilizadas. Lo anterior, se corrobora al observar las diferencias entre los grupos creados según el numero de hijas que aportan al estudio (Cuadro 1), en donde, al incrementar esta cantidad, el promedio de VG y CF de EG-MEX y EG-I se aproximan, y como consecuencia, las $\rho \mathrm{P}$ entre ambas evaluaciones ascienden de manera gradual. Las $\beta$ y sus errores estándar calculados a través de los análisis de regresión, muestran un decremento de aproximadamente la mitad para PL y dos terceras partes para PG y PP, de la EG-MEX con la EG-I (de $23 \pm 0.6 \mathrm{~kg}$ a $42 \pm 0.7 \mathrm{~kg}$ para PL, de $0.3 \pm 0.02 \mathrm{~kg}$ a $0.92 \pm 0.03$ $\mathrm{kg}$ para $\mathrm{PG}$ y de $0.48 \pm 0.02 \mathrm{~kg}$ a $1.1 \pm 0.02 \mathrm{~kg}$ para $\mathrm{PP}$ ), pero el comportamiento de ambas evaluaciones a través de los años es muy similar, diferencia que puede ser explicada por el ajuste en las bases genéticas. Las conclusiones de otro estudio señalan que la información generada en la evaluación internacional es un indicador más preciso en comparación con los resultados obtenidos en hijas evaluadas en un solo país ${ }^{(21)}$, situación que se comprueba en este estudio al encontrar incrementos en las CF para las EG-I en las tres características. 
Figura 1: Tendencia de los valores genéticos (VG) por año de nacimiento de sementales incluidos en la evaluación mexicana (EG-MEX) e internacional (EG-I) para producción de leche (PL), grasa (PG) y proteína (PP) en kilogramos
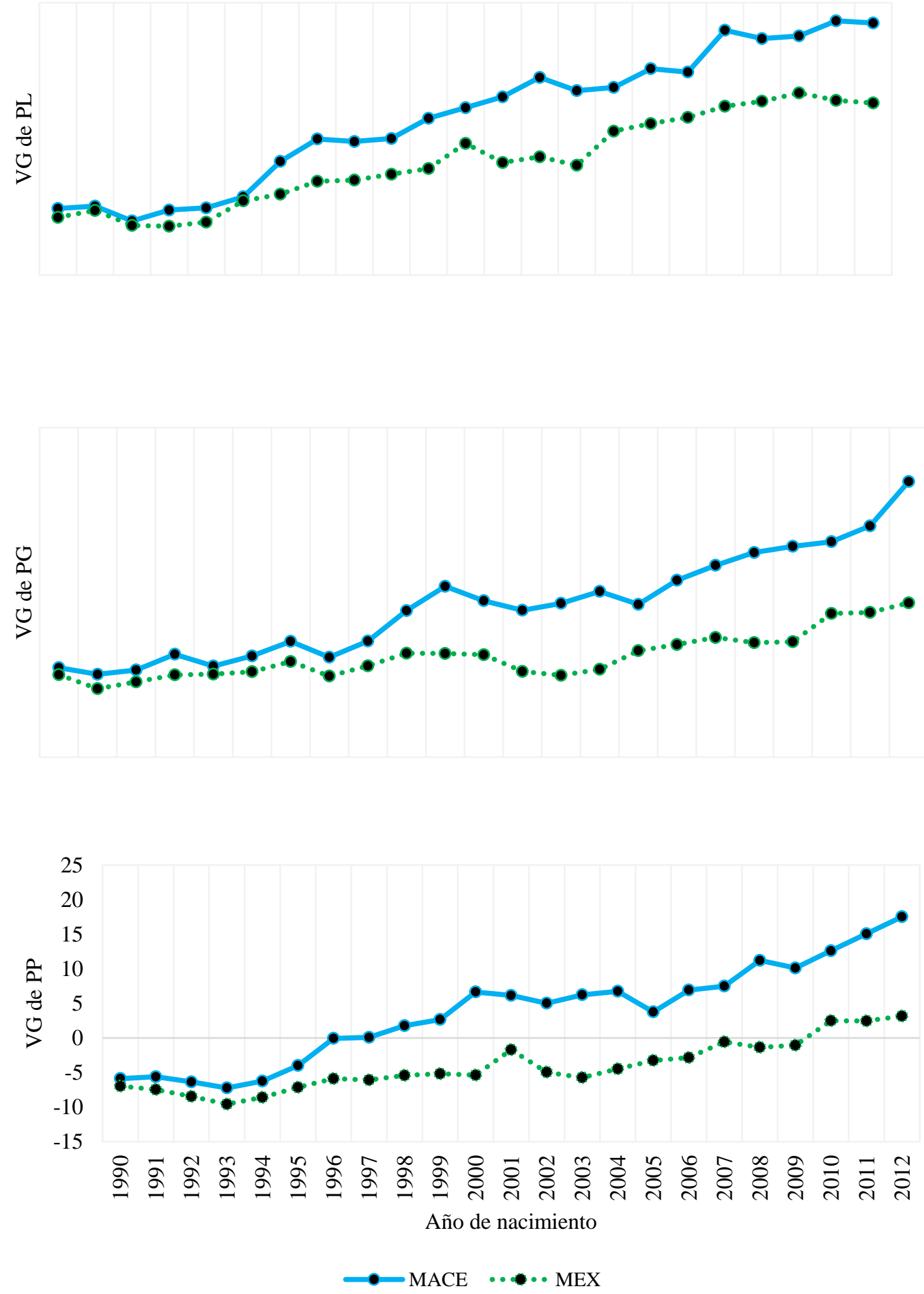
Figura 2: Tendencias de las confiabilidades (CF) por año de nacimiento de sementales incluidos en la evaluación mexicana (EG-MEX) e internacional (EG-I) para producción de leche (PL), grasa (PG) y proteína (PP) en kilogramos
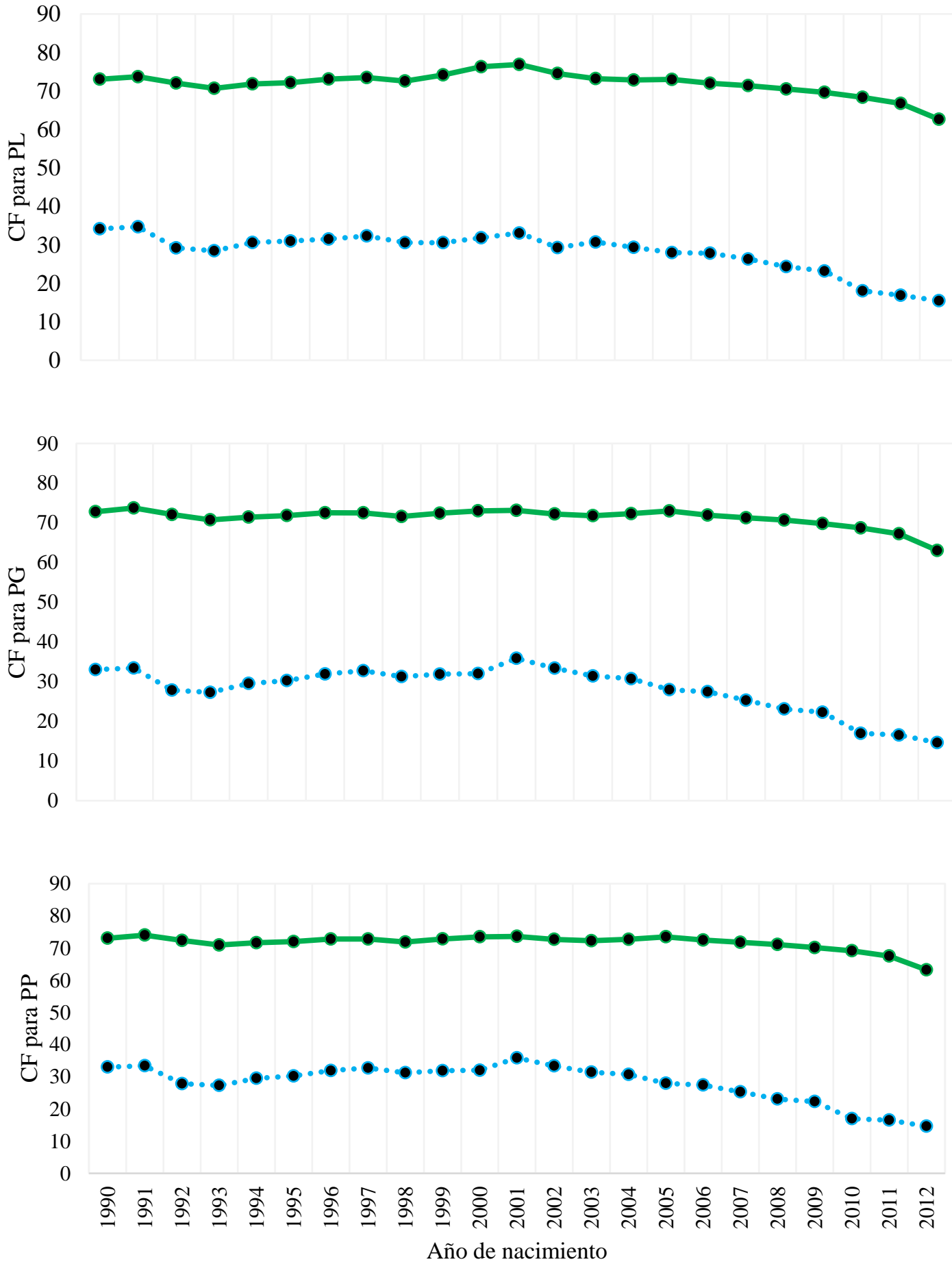

$\longrightarrow$ Confiabilidad MACE $\quad \cdots \bullet$ Confiabilidad MEX 
Cuadro 1: Valores genéticos (VG), confiabilidades (CF) y correlaciones de Pearson $(\rho \mathrm{P})$ para sementales clasificados por el número de hijas en México y participantes en las evaluaciones genéticas mexicanas (EG-MEX) e internacionales (EG-I) para producción de leche $(\mathrm{PL})$, grasa $(\mathrm{PG})$ y proteína $(\mathrm{PP})$ en kilogramos $(P<0.05)$

\begin{tabular}{|c|c|c|c|c|c|c|}
\hline & $\begin{array}{l}\text { VG EG- } \\
\text { MEX }\end{array}$ & $\begin{array}{l}\text { VG } \\
\text { EG-I }\end{array}$ & $\begin{array}{c}\text { CF } \\
\text { EG-MEX }\end{array}$ & $\begin{array}{c}\text { CF } \\
\text { EG-I }\end{array}$ & $\begin{array}{c}\rho P \\
\text { VG }\end{array}$ & $\begin{array}{l}\rho \mathbf{P} \\
\mathbf{C F}\end{array}$ \\
\hline$>50$ & $-2 \pm 325$ & $88 \pm 333$ & $45 \pm 18$ & $86 \pm 11$ & 0.73 & 0.48 \\
\hline PL $>10 y \leq 50$ & $-137 \pm 302$ & $44 \pm 364$ & $31 \pm 9$ & $74 \pm 6$ & 0.52 & 0.28 \\
\hline$\leq 10$ & $-261 \pm 295$ & $-151 \pm 420$ & $26 \pm 7$ & $70 \pm 5$ & 0.61 & 0.19 \\
\hline$>50$ & $0.7 \pm 11.2$ & $4.7 \pm 12.5$ & $56 \pm 18$ & $84 \pm 9$ & 0.66 & 0.62 \\
\hline$P G>10 y \leq 50$ & $-1.1 \pm 9.4$ & $4.1 \pm 12.6$ & $35 \pm 11$ & $76 \pm 5$ & 0.46 & 0.24 \\
\hline$\leq 10$ & $-3.1 \pm 7.2$ & $1.4 \pm 12.4$ & $24 \pm 8$ & $71 \pm 5$ & 0.45 & 0.19 \\
\hline$>50$ & $1.5 \pm 9.1$ & $5.9 \pm 11.2$ & $56 \pm 18$ & $85 \pm 8$ & 0.64 & 0.62 \\
\hline$P P>10 y \leq 50$ & $-1.3 \pm 8.2$ & $5.8 \pm 11.1$ & $35 \pm 11$ & $77 \pm 5$ & 0.42 & 0.22 \\
\hline$\leq 10$ & $-4.7 \pm 7.4$ & $0.9 \pm 12.4$ & $25 \pm 8$ & $71 \pm 6$ & 0.53 & 0.17 \\
\hline
\end{tabular}

Al realizar el primer análisis de reordenamiento de sementales de acuerdo a su VG (EG-MEX y EG-I), se observa que existe un reordenamiento en los sementales comprobado por las bajas $\rho S$ calculadas (0.63 para PL, 0.48 para PG y 0.55 para PP). Esta correlación disminuye su valor al incrementar la $i p_{s}$, siendo el grupo de $10 \%$ superior para las tres características el que mayor reordenamiento presenta, resultando con solamente $35 \%$ de sementales en común. Los resultados de los otros grupos se presentan en el Cuadro 2.

Cuadro 2. Valores genéticos (VG), confiabilidades (CF) y correlaciones de Spearman ( $\rho S$ ) para sementales agrupados por diferentes percentiles $\left(i p_{s}\right)$ y ordenados de manera descendente por la evaluación genética de México (EG-MEX) e internacional (EG-I), con el porcentaje de sementales en común en ambos ordenamientos $(\% \mathrm{Sc})$ para producción de leche $(\mathrm{PL})$, grasa $(\mathrm{PG})$ y proteína (PP) en kilogramos $(P<0.05)$

\begin{tabular}{|c|c|c|c|c|c|c|c|c|c|c|c|c|}
\hline & \multirow[b]{2}{*}{$i p_{s}$} & \multirow[b]{2}{*}{$\begin{array}{l}\% \\
\text { Sc }\end{array}$} & \multicolumn{5}{|c|}{ ORDENADOS POR EG-MEX } & \multicolumn{5}{|c|}{ ORDENADOS POR EG-I } \\
\hline & & & $\begin{array}{c}\text { VG } \\
\text { EG-MEX }\end{array}$ & $\begin{array}{c}\text { VG } \\
\text { EG-I }\end{array}$ & $\begin{array}{c}\text { CF } \\
\text { EG-MEX }\end{array}$ & $\begin{array}{c}\text { CF } \\
\text { EG-I }\end{array}$ & $\begin{array}{r}\rho S \\
\text { VG }\end{array}$ & $\begin{array}{c}\text { VG } \\
\text { EG-MEX }\end{array}$ & $\begin{array}{c}\text { VG } \\
\text { EG-I }\end{array}$ & $\begin{array}{c}\text { CF } \\
\text { EG-MEX }\end{array}$ & $\begin{array}{c}\text { CF } \\
\text { EG-I }\end{array}$ & $\rho S$ VG \\
\hline \multirow{5}{*}{ PL } & 10 & 35 & $356 \pm 148$ & $364 \pm 278$ & $37 \pm 16$ & $79 \pm 11$ & 0.21 & $149 \pm 264$ & $654 \pm 177$ & $28 \pm 14$ & $73 \pm 9$ & * \\
\hline & 20 & 53 & $249 \pm 151$ & $312 \pm 309$ & $34 \pm 15$ & $77 \pm 10$ & 0.23 & $96 \pm 271$ & $504 \pm 199$ & $29 \pm 13$ & $73 \pm 9$ & 0.17 \\
\hline & 30 & 64 & $176 \pm 162$ & $252 \pm 330$ & $32 \pm 14$ & $76 \pm 10$ & 0.33 & $52 \pm 276$ & $402 \pm 218$ & $29 \pm 13$ & $74 \pm 9$ & 0.26 \\
\hline & 40 & 70 & $114 \pm 177$ & $194 \pm 346$ & $32 \pm 14$ & $75 \pm 10$ & 0.39 & $13 \pm 279$ & $319 \pm 238$ & $30 \pm 13$ & $74 \pm 9$ & 0.33 \\
\hline & 50 & 74 & $58 \pm 195$ & $137 \pm 359$ & $31 \pm 13$ & $74 \pm 9$ & 0.48 & $-30 \pm 285$ & $248 \pm 256$ & $30 \pm 13$ & $74 \pm 9$ & 0.43 \\
\hline \multirow{5}{*}{ PG } & 10 & 26 & $11.4 \pm 5.2$ & $12.6 \pm 11.1$ & $35 \pm 17$ & $75 \pm 8$ & 0.18 & $4.1 \pm 8.4$ & $25.5 \pm 5.7$ & $25 \pm 14$ & $73 \pm 7$ & 0.14 \\
\hline & 20 & 42 & $8.4 \pm 5$ & $10.9 \pm 11.5$ & $32 \pm 16$ & $74 \pm 8$ & 0.19 & $3.3 \pm 8.1$ & $20.4 \pm 6.6$ & $26 \pm 14$ & $74 \pm 7$ & 0.14 \\
\hline & 30 & 53 & $6.8 \pm 5.1$ & $9.8 \pm 11.7$ & $31 \pm 15$ & $73 \pm 7$ & 0.21 & $2.3 \pm 7.9$ & $27.1 \pm 13.9$ & $17 \pm 7$ & $74 \pm 7$ & 0.21 \\
\hline & 40 & 58 & $4.7 \pm 5.3$ & $7.7 \pm 11.8$ & $30 \pm 14$ & $73 \pm 7$ & 0.32 & $1.4 \pm 7.9$ & $27.8 \pm 14.1$ & $14 \pm 7$ & $74 \pm 7$ & 0.26 \\
\hline & 50 & 66 & $3.4 \pm 5.5$ & $6.7 \pm 11.8$ & $30 \pm 14$ & $73 \pm 7$ & 0.33 & $0.8 \pm 7.8$ & $28.4 \pm 14.1$ & $12 \pm 8$ & $74 \pm 7$ & 0.27 \\
\hline PP & 10 & 29 & $10.1 \pm 3.5$ & $12.6 \pm 11.1$ & $34 \pm 17$ & $76 \pm 8$ & $*$ & $3.3 \pm 6.9$ & $24.6 \pm 5.4$ & $24 \pm 13$ & $74 \pm 7$ & $*$ \\
\hline
\end{tabular}




\begin{tabular}{cccccccccccc}
\hline 20 & 47 & $7.2 \pm 3.8$ & $10.9 \pm 11.5$ & $32 \pm 16$ & $75 \pm 8$ & 0.16 & $2.4 \pm 7.3$ & $19.9 \pm 6.1$ & $26 \pm 14$ & $74 \pm 7$ & 0.13 \\
30 & 59 & $5.8 \pm 4.1$ & $9.8 \pm 11.7$ & $31 \pm 16$ & $75 \pm 8$ & 0.26 & $1.9 \pm 7.4$ & $16.8 \pm 6.7$ & $27 \pm 14$ & $74 \pm 7$ & 0.16 \\
40 & 63 & $3.7 \pm 4.7$ & $7.7 \pm 11.8$ & $31 \pm 15$ & $74 \pm 7$ & 0.37 & $0.8 \pm 7.6$ & $14.3 \pm 7.3$ & $28 \pm 14$ & $74 \pm 7$ & 0.28 \\
50 & 68 & $2.5 \pm 5.1$ & $6.7 \pm 11.8$ & $30 \pm 15$ & $74 \pm 7$ & 0.44 & $0.2 \pm 7.6$ & $12.1 \pm 7.9$ & $28 \pm 14$ & $75 \pm 7$ & 0.32 \\
\hline
\end{tabular}

En el análisis de $\Delta I_{s}$, al utilizar la $i p_{s}$ mayor (que incluye al percentil más alto), se observó un incremento más grande para las tres evaluaciones (EG-MEX, EG-I y EG-MACE) en las tres características, lo que sugiere el uso de estos sementales para las siguientes generaciones (Cuadro 3); pero habría que tomar en cuenta diversos factores dentro de la población (tasas de consanguinidad, trato preferencial, adaptación a los sistemas de producción, etc.), para optimizar el aprovechamiento de dichos ejemplares. Además, se pudo observar un mayor $\Delta I_{s}$ cuando se usan los resultados de EG-MACE, debido a la mayor disponibilidad de animales, ya que representa una oportunidad para incrementar las $\Delta I_{s}$, incrementar la variabilidad genética y proporcionar mayor cantidad de material de seleccióna los productores; sin embargo, para recomendar su uso, es necesario considerar factores como genotipo ambiente, disponibilidad del germoplasma y la selección para otros caracteres de interés en la población.

Cuadro 3: Superioridad genética esperada por año $\left(\Delta I_{S}\right)$ de los sementales agrupados por diferentes intensidades de selección $\left(i p_{s}\right)$ y ordenados de manera descendente para la evaluación genética en México. Bajo dicho orden, se tomó en cuenta el valor genético estimado en México (MEX), el internacional con hijas en México (I) y a todos aquellos sementales que participaron en la prueba internacional completa y que pueden o no, tener hijas en México (MACE)

\begin{tabular}{ccccc}
\hline & $\boldsymbol{i} \boldsymbol{p}_{\boldsymbol{s}}$ & $\Delta \boldsymbol{I}_{\boldsymbol{s}}$-MEX & $\Delta \boldsymbol{I}_{\boldsymbol{s}}$-I & $\Delta \boldsymbol{I}_{\boldsymbol{s}}-\mathbf{M A C E}$ \\
\hline \multirow{4}{*}{$\mathrm{PL}$} & 10 & 18.5 & 24.1 & 28.5 \\
& 20 & 14.7 & 19.2 & 22.7 \\
& 30 & 11.9 & 15.6 & 18.5 \\
& 40 & 10.2 & 13.3 & 15.7 \\
& 50 & 8.4 & 10.9 & 12.9 \\
$\mathrm{PG}$ & 10 & 0.55 & 0.84 & 0.90 \\
& 20 & 0.44 & 0.67 & 0.72 \\
& 30 & 0.36 & 0.55 & 0.58 \\
& 40 & 0.30 & 0.46 & 0.50 \\
$\mathrm{PP}$ & 50 & 0.25 & 0.38 & 0.41 \\
& 10 & 0.54 & 0.83 & 1.83 \\
& 20 & 0.43 & 0.66 & 1.46 \\
& 30 & 0.35 & 0.54 & 1.19 \\
& 40 & 0.30 & 0.46 & 1.01 \\
& 50 & 0.25 & 0.38 & 0.83 \\
\hline
\end{tabular}


Otra manera de evaluar la similitud entre los diferentes sistemas de evaluación, es por medio de las CGI entre las EG-MEX y los países participantes en las EG-I, en donde el promedio entre EG-MEX y los 29 países participantes fue de $0.79 \pm 0.04$ para PL, de $0.80 \pm 0.04$ para PG y de $0.81 \pm 0.04$ para PP. Las CGI con los países con los que México tiene mayor intercambio de germoplasma y que fueron calculadas con base en más de 200 sementales, como lo son Estados Unidos de América, Canadá, Países Bajos, Alemania-Austria, Gran Bretaña, Italia y Francia, alcanzaron niveles medios (0.80 aproximadamente), mientras que, las correlaciones más altas se observaron con con Israel, Eslovenia, Estonia, Letonia y Lituania (alcanzando CGI de 0.85, 0.86 y 0.87, para PL, PG y PP respectivamente), lo que pudiera estar explicado por el bajo número de sementales en común ( $<50$ sementales) y su la alta preferencia que se encuentra corroborado por el alto número de hijas ( $>20$ hijas) . Las CGI más bajas resultaron de países en los que predominan sistemas de producción diferentes a los encontrados en México, como son: Nueva Zelanda, Australia e Irlanda con valores de 0.69 para PL y de 0.7 para PG y PP). Las diferencias observadas entre las CGI en los diferentes países, podría estar explicada por la adaptación del semental a un país en específico y no a las diferencias en los modelos o trato preferencial de los sementales ${ }^{(21)}$, por lo que, si esta aseveración es correcta, las estimaciones de los VG de los sementales utilizados a nivel nacional, serán más aproximadas a las obtenidas en países con las condiciones de producción similares a las de México. Lo anterior podría explicar los resultados obtenidos con países que contribuyen en gran proporción al número de hijas en México, como Estados Unidos de América, Canadá y los Países Bajos, pero que no tienen las CGI más altas con México. Estos países presentan diferencias con México en las condiciones de producción, la definición de las bases genéticas, los diferentes índices de heredabilidad $\left(\mathrm{h}^{2}\right)$ estimados por cada país, y en la complejidad de los modelos utilizados para las estimaciones de los VG, así como los efectos aplicados en cada uno de ellos. Por lo anterior, es recomendable analizar a los grupos integrados por países con condiciones de evaluación semejantes; y así ampliar las expectativas de mercado internacional e intercambio de información de manera eficiente.

Una área de oportunidad para los países que son miembros de Interbull, es el uso e intercambio de información genómica, que puede traer importantes beneficios a los sistemas de evaluaciones genéticas, como por ejemplo, realizar una predicción más efectiva y rápida de las interacciones genéticas ${ }^{(22)}$, la disminución en el tiempo de comercialización del material genético, refiriéndose a características de importancia reproductiva y de consanguinidad $^{(23)}$ y que en conjunto, se ve reflejado en el aumento de la precisión de los VG y de sus $\mathrm{CF}^{(24)}$. 


\section{Conclusiones e implicaciones}

El impacto de la información extranjera en la EG-MEX es positivo, debido a que mejora la CF de los VG de los sementales usados en México, sobre todo de aquellos que tienen una cantidad limitada de hijas en nuestro país, por lo que se recomienda continuar con la participación en el programa de Interbull. En este estudio, al realizar el ordenamiento de los sementales y al verificar la superiordidad genética por año, se pudieron observar diferencias importantes de los valores genéticos entre las evaluaciones, generando una oportunidad de mejoramiento en la población Holstein mexicana. Los sementales no usados en México y que tienen un alto potencial genético para las características evaluadas, representan una oportunidad de mejoramiento en la población; por lo que, es importante realizar su identificación, valoración y posible incorporación en la población Holstein de nuestro país. Los resultados obtenidos al incorporar la EG-MEX a la EG-I, muestran las diferencias entre diversos países participantes (explicadas por los diversos sistemas de producción, factores ambientales, etc.), por lo que es primordial considerar el uso de la información validada de manera internacional en los procesos de selección de animales productores de leche y sus componentes de la raza Holstein en México, ya que contribuirá de manera importante al incremento del progreso genético, productivo y reproductivo de la población.

\section{Agradecimientos}

Al INIFAP-CENIDFyMA por su apoyo y financiamiento para la realización de este trabajo, a través del proyecto "Estudio de la consanguinidad y su efecto sobre características productivas y reproductivas en ganado Holstein" SIGI : 11513634465 y a la Facultad de Ciencias Naturales de la Universidad Autónoma de Querétaro por el apoyo y financiamiento parcial del trabajo a través del proyecto "Desarrollo de evaluaciones genéticas internacionales de ganado Holstein en México".

\section{Conflicto de intereses}

Los autores declaran que no existen conflictos de interés.

\section{Literatura citada:}

1. Ruiz-López FJ, García-Ruiz A, Martínez-Marín GJ. ¿Qué Toro? Evaluación genética de toros y vacas Holstein para producción de leche, conformación y longevidad. Centro Nacional de Investigación Disciplinaria en Fisiología y Mejoramiento Animal, INIFAPSAGARPA y Asociación Holstein de México. Libro Técnico No. 58, Colón, Querétaro. 2018. 
2. Moro-Méndez J, Ruiz-López FJ. Mejoramiento genético de características de conformación en ganado Holstein. Vet Méx 1998;9(4):385-398.

3. REDGATRO. Red de Investigación e Innovación Tecnológica para la Ganadería Bovina Tropical. Estado del arte sobre investigación e innovación tecnológica en ganadería bovina tropical. Consejo Nacional de Ciencia y Tecnología. Libro Técnico No. 4, Ciudad de México. 2015.

4. VanRaden PM, Wiggans GR. Derivation, calculation, and use of national animal model information. J Dairy Sci 1991;74(8):2737-2746.

5. Schaeffer LR, Zhang W, Robinson A, Chesnais J, Wilmink H, Wiggans G, et al. Multiple trait across country evaluation of dairy sires. Interbull Bulletin 1993:1-21.

6. Mäntysaari EA, Liu Z, VanRaden PM. Interbull validation test for genomic evaluations. Interbull Bulletin 2010; 41:17-21.

7. INTERBULL. International Bull Evaluation Service. Code of practice for the international genetic/genomic evaluation of dairy bulls at the Interbull Centre. DABG-ICAR. 2017.

8. Cameron ND. Selection indices and prediction of genetic merit in animal breeding. 1st ed. UK: CAB International; 1997.

9. Misztal I, Tsuruta S, Strabel T, Auvray B, Druet T, Lee DH. BLUPF90 and related programs (BGF90). In: CD-ROM communication, Proceedings of the 7th World congress on genetics applied to livestock production. Montpellier, France. 2002:7-28.

10. Misztal I. Computational techniques in animal breeding. 1st ed. USA: University of Georgia; 2000.

11. Lynch M, Walsh B. Genetics and analysis of quantitative traits. 1st ed. USA: Sinauer Associates; 1998.

12. Gilmour AR, Gogel BJ, Cullis BR, Thompson R. ASReml User Guide. Relase 3.0. UK: VSN International Ltd; 2009.

13. Fikse WF, Banos G. Weighting factors in international genetic evaluations: effects on international breeding values and reliability estimates. Interbull Bulletin 1999;(22):1-6.

14. Schaeffer LR. Multiple-country comparison of dairy sires. J Dairy Sci 1994;77(9):26712678.

15. Schaeffer LR. Multiple trait animal models. Technical notes, Univ. Guelph 1999; (15) 113. http://www.aps.ouguelph.ca/ 1rs/Animalz/lesson15. Accessed 6 Apr, 2019.

16. Bourdon RM. Understanding animal breeding. 2nd ed. USA: Prentice Hall; 2000. 
17. Schaeffer LR. Strategy for applying genome-wide selection in dairy cattle. J Anim Breed Genet 2006;123(4):218-223.

18. Powell RL, Wiggans GR, VanRaden PM. Factors affecting calculation and use of conversion equations for genetic merit of dairy bulls. J Dairy Sci 1994;77(9):2679-2686.

19. Miglior F, Muir BL, Van Doormaal BJ. Selection indices in Holstein cattle of various countries. J Dairy Sci 2005;88(3):1255-1263.

20. Benhajali H, Jakobsen J, Mattalia S, Ducrocq V. Illustration of an international genetic evaluation robust to inconsistencies of genetic trends in national evaluation. Interbull Bulletin 2013;(47):82-89.

21. Jones LP. A simple approximation to the reliability of Interbull proofs for foreign bulls. Interbull Bulletin 1997;(16):13-15.

22. VanRaden PM, Sullivan PG. International genomic evaluation methods for dairy cattle. Genetics Selection Evolution 2010;42(1):7-15.

23. Hayes BJ, Daetwler HD, Bowman P, Moser G, Tier B, Crump R, et al. Accuracy of genomic selection: Comparing theory and results. Association for the Advancement of Animal Breeding and 30th Anniversary Conference. Proc Assoc Advmt Animal Breed 2009;(17):352-355.

24. Lund MS, de Roos AP, de Vries AG, Druet T, Ducrocq V, Fritz S, et al. A common reference population from four European Holstein populations increases reliability of genomic predictions. Genet Sel Evol 2011;43(1):43-50. 